\title{
Coronavirus Replication, Transcription, and RNA Recombination
}

\author{
Robbert G. VAn der Most and Willy J. M. SpaAN
}

\section{INTRODUCTION}

With the advent of recombinant DNA technology, our knowledge of the transcription and replication processes of positive-strand RNA viruses has increased profoundly. A major breakthrough in this field has been the development of full-length cDNA clones from which infectious RNA transcripts can be made. Such clones have now been constructed for members of the Picorna(Racaniello and Baltimore, 1981), Bromo- (Ahlquist et al., 1984), Alpha- (Rice et al., 1987) and Flaviviridae (Lai et al., 1991; Rice et al., 1989). Unfortunately, the enormous length of the coronavirus genome has hampered the construction of a full-length cDNA clone so far.

In this chapter we will address new data that have been published in the field of coronavirus RNA replication, transcription, and recombination. In addition, we will discuss how cDNA clones of defective interfering (DI) RNAs may provide a promising alternative to study the replication and transcription of coronaviruses. Previous reviews that dealt with coronavirus transcription and replication appeared in 1988 (Spaan et al., 1988) and 1990 (Lai, 1990).

ROBBERT G. VAN DER MOST AND WILLY J. M. SPAAN - Department of Virology, Institute of Medical Microbiology, Leiden University, 2300 AH Leiden, The Netherlands.

The Coronaviridae, edited by Stuart G. Siddell, Plenum Press, New York, 1995. 


\section{REPLICATION}

Coronaviruses are positive-strand RNA viruses. Thus, upon infection, the genomic RNA is translated to produce the RNA-dependent RNA polymerase. The RNA polymerase transcribes the viral genome into a complementary RNA. The negative-stranded RNA in turn serves as a template for the synthesis of genomic RNA. The viral and/or host enzymes involved in the RNA replication process of coronaviruses are unknown. The translation product(s) of the huge polymerase gene are proteolytically processed; however, the processing pathway is not well characterized (Baker et al., 1989, 1993; Denison and Perlman, 1987; Denison et al., 1991, 1992; Weiss et al., 1994). By analogy with other positive-strand RNA viruses (Andino et al., 1993; Lemm and Rice, 1993a,b), it is thought that processed end-products as well as precursor polypeptides and host factors may be involved in the replication process.

The RNA polymerase must recognize specific signals that control the initiation of plus- and minus-strand RNA synthesis. Mapping of these cisacting signals is a prerequisite for our understanding of coronavirus replication. Because a full-length cDNA clone of a coronavirus is not available, DI RNAs are the tools of choice to study these signals. DI RNAs are replicated in virus-infected cells, and must therefore contain the essential cis-acting replication signals.

During the last few years several natural DI RNAs of murine hepatitis virus (MHV) strains JHM (Makino et al., 1985, 1988a, 1990; Makino and Lai, 1989a) and A59 (van der Most et al., 1991) have been characterized and used as templates to construct full-length cDNA clones. Synthetic DI RNAs can be transcribed in vitro from these clones and are replicated on transfection into MHVinfected cells. The structures of the JHM DIs DIssE and DIssF and the A59 DI MIDI are schematically shown in Fig. 1. All three DI RNAs contain the $5^{\prime}$ and $3^{\prime}$ terminal sequences of the viral genome. MIDI is a synthetic copy of the natural DI RNA DI-a and consists of three noncontiguous fragments of the viral genome, i.e., the $5^{\prime}$ terminal 3889 nucleotides (nt), 799 nt derived from the $3^{\prime}$ end of open reading frame $1 \mathrm{~b}(\mathrm{ORFlb})$, and the $3^{\prime}$ terminal $806 \mathrm{nt}$ (van der Most et al., 1991). Both DIssF and MIDI contain sequences from the 3' end of ORFIb, which are required for RNA packaging (Makino et al., 1990; van der Most et al., 1991). This packaging signal was mapped as a $61 \mathrm{nt}$ sequence that could be folded into a bulged stem-loop structure (Fosmire et al., 1992). It is not yet known whether this sequence alone is sufficient for RNA packaging.

The minimal sequence requirements for replication can be mapped by comparing the sequences of the three natural DI RNAs and by deletion mutagenesis. Deletion mapping of DIssE, DIssF, and MIDI has demonstrated that the $3^{\prime}$ replication signal comprises the $3^{\prime}$ terminal $447 \mathrm{nt}$ (Kim et al., 1993a; Lin and Lai, 1993; van der Most and Spaan, unpublished results) (Fig. 1). It has been suggested that a short conserved sequence element in the $3^{\prime}$ nontranslated region of the genomic RNA (5'-CGAAGAGC-3') acts as a replication signal for minus-strand synthesis (Lai, 1990). Clearly, the results from the deletion mapping suggest that this sequence alone is not sufficient and that the signal(s) comprise a larger stretch of sequences. However, very recently Lin et al. have provided evidence that the $3^{\prime}$ terminal 55 nucleotides of the positive strand RNA plus the poly(A) tail comprise the cis-acting signal for the initiation of 


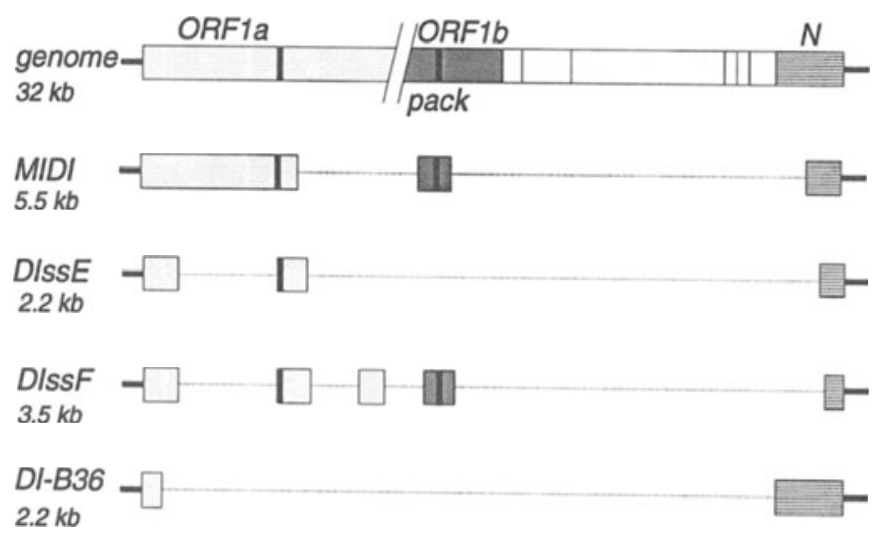

\section{$0 \quad 1234 \mathrm{~kb}$}

FIGURE 1. Schematic structure of the genomes of MIDI, DIssE, DIssF, and B36. The sequences derived from ORFla, ORFlb, and the $3^{\prime}$ end of the genome (N) are indicated by different shadings. The putative enhancer sequence in ORFla and the RNA packaging signal in ORFlb (pack) are indicated by black bars.

minus-strand synthesis (Lin et al., 1994). This would imply that part of the $3^{\prime}$ terminal $447 \mathrm{nt}$ that were identified as the $3^{\prime}$ replication signal, are in fact required for positive strand synthesis (Lin et al., 1994). Thus, initiation of positive strand synthesis could involve interactions between $5^{\prime}$ - and $3^{\prime}$-terminal signals.

Mapping of the $5^{\prime}$ replication signal has yielded conflicting results on two points. First, it is unclear which sequences at the very $5^{\prime}$ terminus of the genome are needed. An artificial MHV-A59 DI RNA, DI-B36, which consists of the $5^{\prime}$ terminal $467 \mathrm{nt}$ and the $3^{\prime}$ terminal $1671 \mathrm{nt}$ (Fig. 1), is replicated in MHVA59-infected cells (Masters et a1., 1994). DI-B36 was originally constructed on the basis of a bovine coronavirus (BCV) DI RNA (Hofmann et al., 1990; Masters et al., 1994), which contains the 5' terminal $498 \mathrm{nt}$ and which is also replicated on transfection into BCV-infected cells (Brian et al., 1994). These data suggest that the $5^{\prime} 467 \mathrm{nt}$ comprise the $5^{\prime}$ replication signal. Accordingly, the $5^{\prime}$ terminal $474 \mathrm{nt}$ were found to be sufficient for replication of DIssE (Kim et al., 1993a). In contrast, the $5^{\prime}$ terminal $482 \mathrm{nt}$ do not support replication of DIssF /Lin and Lai, 1993). Deletion of the sequence between positions 482 and 706 abrogates replication of DIssF (Lin and Lai, 1993), whereas deletion of the same fragment from DIssE only reduces replication efficiency slightly (Kim et al., 1993a). Preliminary evidence from our laboratory suggests that for (efficient) replication of the MIDI-derivatives the $5^{\prime} 1.1 \mathrm{~kb}$ is essential (Luytjes, Gerritsma, and Spaan, unpublished results). It is possible that these differences reflect the different experimental protocols used. The deletion mapping experiments with DIssF and MIDI have involved in vivo transcription of DI RNA by a vaccinia virus-encoded T7 RNA polymerase (Fuerst et al., 1986), in combination with DNA transfection (Lin and Lai, 1993; van der Most et al., 1992). Replication of 
the DIssE mutants has been assessed in MHV-infected and RNA-transfected cells (Kim et al., 1993a) and the analysis of DI-B36 has been done with a yet different protocol, which involves seeding of RNA-transfected L cells onto a monolayer of $17 \mathrm{ClI}$ cells (Masters et al., 1994).

The second issue on which the current data conflict is the finding that replication of the JHM DIs DIssE and DIssF requires an internal sequence element of $135 \mathrm{nt}$ (Kim et al., 1993a; Lin and Lai, 1993) (Fig. 1). This sequence element is located $3 \mathrm{~kb}$ from the $5^{\prime}$ terminus of the genome and is contained in MIDI, but is not present in most of its derivatives [e.g., $\Delta \mathrm{H}$-in (de Groot et al., 1992)], nor in DI-B36 (Masters et al., 1994). These DI RNAs are, however, replicated, indicating that this presumptive signal is not an absolute requirement for MHV DI replication. It has been suggested that this signal functions as a replication enhancer (Kim et al., 1993a), which would explain why RNA replication is readily detected in DIssE-transfected and virus-infected cells. Clearly, the precise sequence of the cis-acting signals, the extent to which these data can be extrapolated to the viral genome, and the role of the putative enhancer remain to be elucidated. The sequence elements that have been mapped comprise the $5^{\prime}$ and $3^{\prime}$ nontranslated regions of the genome, as well as parts of ORFla and the $\mathrm{N}$ gene. To date, it is unclear which proteins interact with these replication signals. Several host factors have been shown to bind to the $5^{\prime}$ terminus of the plus-strand and to the $3^{\prime}$ terminus of the minus-strand RNA (Furuya and Lai, 1993), and it is possible that these proteins play a role in the initiation of RNA synthesis. Further characterization of the enzymology of replication will await the reconstruction of a functional polymerase gene or the development of an in vitro RNA replication system.

A peculiar feature of the MHV DI RNAs is that they contain large ORFs. For instance, the three fragments of DIssE as well as the three fragments of MIDI are joined in-frame (Makino et al., 1988a; van der Most et al., 1991). There is now substantial evidence that the presence of large ORFs in the genomes of MHV DI particles is biologically significant (de Groot et al., 1992; Kim et al., 1993b). For DIssE it appears that the ORF is not required in DI-transfected and MHV-infected cells, but is important after one passage (Kim et al., 1993b). In principle, the requirement for a large ORF could be related to RNA stability (de Groot et al., 1992), replication, packaging, or uncoating. The results obtained for DIssE may suggest that the large ORF is not required for RNA replication. However, these data have not yet been confirmed for MIDI and its derivatives (van der Most and Spaan, unpublished results). Furthermore, it is not known if these data can be extrapolated to the viral genomic RNA.

\section{TRANSCRIPTION}

\section{A. Introduction}

Coronavirus gene expression involves the synthesis of five to eight subgenomic mRNAs (Lai, 1990; Spaan et al., 1988). These mRNAs are synthesized in different but constant molar ratios in the infected cell. As an illustration, 
TABLE I. MHV Subgenomic mRNAs, Coding Assignments, and Relative Molarities

\begin{tabular}{llc}
\hline MHV mRNA & \multicolumn{1}{c}{ Protein } & $\begin{array}{c}\text { Relative molarity } \\
\text { (percent) }\end{array}$ \\
\hline mRNA1 (genome) & RNA-dependent RNA polymerase & 10.4 \\
mRNA2 & Nonstructural protein 2a & 4.7 \\
mRNA2-1 (not all strains) & HE glycoprotein & 4.2 \\
mRNA3 & Spike glycoprotein & 6.5 \\
mRNA4 & Nonstructural protein & 13.1 \\
mRNA5 & ORF5a: nostructural protein & 15.7 \\
mRNA6 & ORF5b: small membrane protein & 45.5 \\
mRNA7 & Membrane glycoprotein & \\
\hline
\end{tabular}

${ }^{a}$ From Jacobs et al. (1981).

Table I summarizes the MHV mRNAs, their translation products, and their molar ratios. The mRNAs form a $3^{\prime}$ coterminal nested set, i.e., they have identical $3^{\prime}$ ends but extend for different lengths in the $5^{\prime}$ direction (illustrated for MHV in Fig. 2). Only the $5^{\prime}$ most gene(s), which is not present in the next smaller mRNA, is translated (Lai, 1990; Spaan et al., 1988) (see Chapter 3). On the genome, the transcription units are preceded by short sequence elements, termed intergenic regions. The complementary intergenic regions on the negative-stranded RNA are thought to function as promoters and will therefore be referred to as the "intergenic promoter sequences" or "promoters" in this chapter.

The subgenomic mRNAs not only form a 3' coterminal nested set, but are also 5' coterminal: all mRNAs contain a common 5 ' leader sequence (Lai et al., 1984; Spaan et al., 1983) (Fig. 2). For different coronaviruses, the length of the leader ranges from 65 to $98 \mathrm{nt}$. The leader sequence is only found at the very $5^{\prime}$ terminus of the genome, which implies that the synthesis of subgenomic mRNAs involves fusion of noncontiguous sequences. To explain the synthesis of leader-containing subgenomic mRNAs, several models have been put forward. For instance, it has been suggested that the mRNAs arise via splicing of larger precursor RNAs or by "looping-out" of intervening sequences. The socalled leader-primed transcription model proposes that short leader RNAs are transcribed from the $3^{\prime}$ end of the genomic negative strand and act as primers for subgenomic mRNA synthesis (Lai et al., 1984; Spaan et al., 1983). Because in earlier experiments, only genome-length negative-strands were found in MHVinfected cells (Lai et al., 1982), it seemed that these molecules served as the exclusive templates for transcription. However, the recent discovery of subgenomic negative-strands and double-stranded subgenomic RNAs in coronavirus-infected cells has provoked renewed discussion (Hofmann et al., 1990; Sawicki and Sawicki, 1990; Sethna et al., 1989|: these findings imply that the subgenomic mRNAs could also be transcribed from their negative-stranded counterparts.

Much of our knowledge of coronavirus transcription has been obtained by 


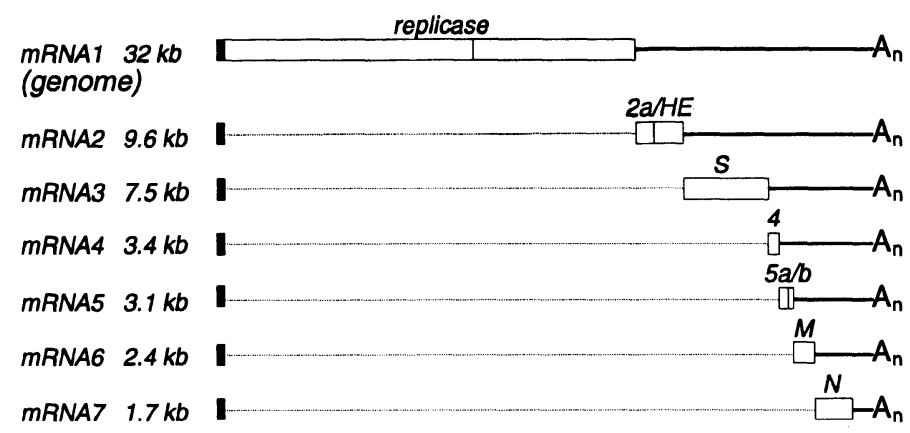

FIGURE 2. Coding assignments and the nested set structure of the MHV subgenomic mRNAs. The common $5^{\prime}$ leader sequence is indicated by a black box. The $5^{\prime}$ most unique ORF(s) of each mRNA are indicated by open boxes. The sequences downstream of the leader of the subgenomic mRNAs will be referred to as "body" sequences.

sequencing subgenomic mRNAs and intergenic regions and by analyzing negative-stranded RNAs. Furthermore, an experimental system has been set up based on synthetic DI RNAs carrying intergenic promoter sequences (Makino et a1., 1991; van der Most et al., 1994). In the present situation, two basic questions can be asked. How are the leader sequences attached to the downstream "body" sequences and which are the templates for mRNA synthesis? The next sections will address the experimental evidence that has accumulated on these issues. We will focus on the discovery of the subgenomic negative strands and its consequences for the current coronavirus transcription models. We will also discuss the role and the sequence requirements of the intergenic regions and the role of basepairing in determining mRNA abundance.

\section{B. Negative-Stranded RNAs}

The actual double-stranded structure that "produces" RNA is designated the replicative intermediate (RI). Visualization of double-stranded RNAs by gel electrophoresis and fluorography usually involves digestion of the excess single-stranded RNA by RNase A. The remaining double-stranded RNaseresistant molecules are referred to as the replicative forms (RFs). The RFs do not always correspond to the RIs because the RIs could also have been digested at certain RNase-sensitive sites to yield smaller RFs.

In 1989, Sethna and co-workers reported the discovery of genomic and subgenomic negative-stranded RNAs in transmissible gastroenteritis virus (TGEV)-infected cells. They identified the negative-stranded RNAs by using strand-specific oligonucleotide probes in Northern (RNA) blot analyses. This observation was confirmed for BCV (Hofmann et al., 1990). It was subsequently demonstrated that the subgenomic negative strands contained an anti-leader sequence (Sethna et al., 1991).

RNase digestion of cytoplasmic RNA isolated from TGEV-infected cells yielded a set of RFs corresponding to the subgenomic mRNAs (Sethna et al., 
1989|. The RFs contained very small quantities of full-length positive-stranded RNAs, whereas full-length negative-stranded RNAs were abundant. This suggested that positive-stranded RNAs were being synthesized from a negativestrand template. However, these authors did not provide evidence that the subgenomic RFs did in fact correspond to subgenomic RIs. In 1990, Sawicki and Sawicki reported the existence of both genomic and subgenomic RFs in MHVinfected cells. They showed that each subgenomic RF was derived from a corresponding subgenomic RI. The RIs were transcriptionally active: following a very short pulse $(2 \mathrm{~min})$ with $\left[{ }^{3} \mathrm{H}\right]$ uridine, the label was predominantly incorporated into the RIs and to a much lesser extent into the subgenomic RNAs. After longer pulse periods (up to $300 \mathrm{~min}$ ), only labeled subgenomic mRNAs could be detected. This was interpreted as an indication that the label "flows through" the RIs into the single-stranded RNAs produced from them (Sawicki and Sawicki, 1990).

The discovery of subgenomic negative strands has led to two different models of how coronaviruses produce their mRNAs, which differ in the nature of the template(s) for mRNA synthesis. Sethna and co-workers speculated that the subgenomic mRNAs might in fact be amplified as independent replicons (Fig. 3A). The initial subgenomic mRNA templates for replication could either be present in the virion or could be synthesized from the antigenome via leaderprimed transcription (Sethna et al., 1989). This model implies that, at least early in infection, the genomic RI would yield subgenomic RFs upon RNase digestion (Sawicki and Sawicki, 1990). Sawicki and Sawicki could not detect such RFs and therefore proposed an alternative model. They suggested that subgenomic negative strands transcribed from the genome serve as templates for the synthesis of the corresponding mRNAs and not vice versa (Fig. 3B). To date, all attempts to obtain direct evidence for mRNA replication by transfecting synthetic subgenomic RNAs into coronavirus-infected cells failed to demonstrate any such replication (Brian et al., 1994; Makino et al., 1991; Luytjes and Spaan, unpublished results). Thus, several lines of evidence seem to suggest that the mRNAs may not undergo replication; instead, the hypothesis that the subgenomic mRNAs are transcribed from the subgenomic negative strands has gained some momentum (Sawicki and Sawicki, 1990; Hofmann et al., 1993). Alternatively, it has been argued that the negative strands are synthesized from the subgenomic mRNAs as dead-end products (Jeong and Makino, 1992). It should be noted, however, that these conclusions are based on negative results. For instance, the failure to directly detect mRNA replication could also be explained by assuming that transfected subgenomic RNAs are, for some unknown reason, not suitable templates for replication.

Very recently, Schaad and Baric (1994) have provided strong genetic evidence indicating that, at least late in infection, the subgenomic negative strand RNAs are the functional templates for subgenomic mRNA synthesis. They studied a temperature-sensitive MHV-mutant, defective in negative strand synthesis, and found that the subgenomic RFs still incorporated radiolabel after a shift to the restrictive temperature. Because minus-strand synthesis was blocked, these results strongly suggest that the transcription indeed occurs on subgenomic negative strands (Schaad and Baric, 1994). 
A

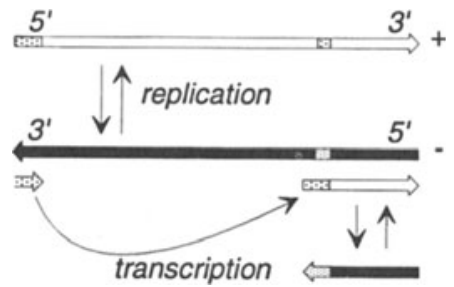

B

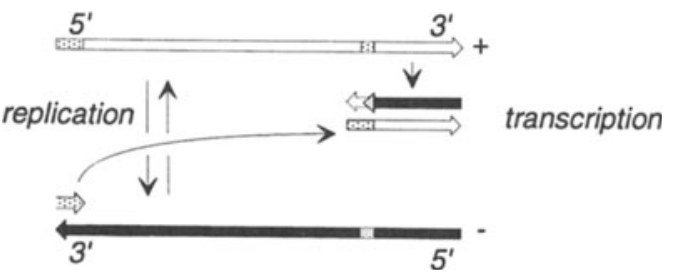

C

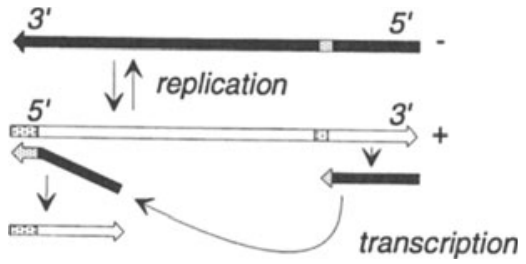

FIGURE 3. Schematic representation of three transcription models. Plus- and minus-strands are indicated by open and by black bars, respectively. On the plus-strands, the leader sequences and intergenic regions are indicated by shading; on the minus-strand, the intergenic promoter is shown by shading. (A) A genomic minus-strand is the template for subgenomic mRNA synthesis. The subgenomic mRNAs are first synthesized by leader-primed transcription and are then amplified. (B) Subgenomic minus-strands are synthesized by premature termination of transcription and serve as templates for leader-primed transcription. (C) A subgenomic minus-strand is synthesized by discontinuous transcription, i.e., the template jump occurs during minus-strand synthesis. The subgenomic minus-strand are the templates for subgenomic mRNA synthesis.

\section{Leader-Primed Transcription}

The experiments described above do not address the question of how the leader sequences are attached to the body sequences. According to the original model of leader-primed transcription, short leader RNAs are transcribed from the $3^{\prime}$ end of the genomic negative strand, translocated to the intergenic promoters on the negative strand(s), and then extended (Lai, 1990; Spaan et al., 1988). A key observation that has been interpreted in favor of this model is that the leader contains a complementary promoter sequence (CPS) near its undetermined $3^{\prime}$ end. This would allow basepairing to occur between the $3^{\prime}$ end of the leader and the promoter, thus facilitating the priming event (Budzilowicz et al., 1985; Spaan et al., 1983).

Before the discovery of subgenomic negative strands, alternative transcription models, e.g., cis-splicing of genome-length precursor RNAs, could be excluded on the basis of the UV inactivation kinetics of mRNAs (Jacobs et al., 
1981; Stern and Sefton, 1982). UV inactivation studies demonstrated that, late in infection, the UV target size of each mRNA corresponds to its physical size. This indicated that transcription of the mRNAs was independent of the synthesis of a genome length precursor. Based on the conclusion that subgenomic negative strands did not exist (Lai et al., 1982), these data provided strong support for the leader-primed transcription model. The discovery of the subgenomic negative strands radically changed the picture, because alternative models could no longer be ruled out. For instance, any model in which mRNAs are transcribed from subgenomic templates is consistent with the UV transcription mapping.

One variant leader-primed transcription model, based on the existence of transcriptionally active subgenomic RIs, is that a nested set of subgenomic negative strands is synthesized first (Fig. $3 \mathrm{~b}$ ). The intergenic promoter sequences act as transcription attenuators in this model. These RNAs then serve as templates for leader-primed transcription (Sawicki and Sawicki, 1990). Thus, leader priming and transcription from subgenomic negative strands are not mutually exclusive. However, this model still assumes the existence of free leader RNAs. There are some indications that free leader transcripts do indeed exist. Baric and co-workers $(1985,1987)$ detected short RNA molecules containing leader sequences in MHV-infected cells. The biological significance of the small RNAs is not clear, though. Baker and Lai (1990) subsequently demonstrated that synthetic leader transcripts are incorporated into subgenomic mRNAs when added to an in vitro transcription system. An important observation was that the CPS at the $3^{\prime}$ of the leader was essential. When the CPS was deleted, the exogenous leaders were not incorporated. Although these data are suggestive, it cannot completely be ruled out that the synthetic leader RNAs did not actually prime transcription but merely functioned as templates for RNA recombination (Liao and Lai, 1992). The high-frequency leader reassortment that occurs during mixed infection of MHV strains has also been interpreted in support of the leader-primed transcription model (Jeong and Makino, 1994; Makino et al., 1986a; Makino and Lai, 1989a; Zhang et al., 1991). However, leader reassortment does not prove leader priming, but rather indicates that there is at least one step in trans in mRNA synthesis. If, for instance, the discontinuous transcription step occurs during negative-strand synthesis (and not by leader priming) (Fig. 3c), one would still observe "leader reassortment" when the (reassorted) negative strands are transcribed to produce mRNAs. Interestingly, leader reassortment appears to depend on a $9 \mathrm{nt}$ sequence directly downstream of the leader; deletion of this sequence results in an exclusive use of the helper virus leader, which is provided in trans (Makino and Lai, 1989a; Zhang et al., 1994). The function of the 9 nt sequence is unknown, but it has been proposed that it functions as a transcriptional terminator, controlling the synthesis of free leader transcripts (Zhang et al., 1994).

As discussed in Section B, there is now genetic evidence strongly suggesting that subgenomic minus-strands are the functional templates for subgenomic mRNA synthesis (Schaad and Baric, 1994). Indeed, one experimental approach to determine whether leader-primed transcription occurs on sub- 
genomic negative strands would be to transfect subgenomic negative strands and determine whether subgenomic mRNAs are transcribed from these templates.

\section{The Intergenic Promoter Sequence}

The intergenic regions are short sequence elements upstream of the transcription units. Because the leader-mRNA junction occurs within the intergenic region, this sequence or its minus-sense counterpart, the intergenic promoter sequence, is considered to be crucial for mRNA synthesis. The potential basepairing between the leader CPS and the promoter is one of the cornerstones of the leader-primed transcription model. The intergenic promoter sequences for different genes in one coronavirus may differ slightly in sequence and in the extent of potential basepairing with the leader CPS. For MHV, the extent of potential basepairing ranges from 9 to 18 basepairs; every promoter contains the sequence $3^{\prime}$ UUAGAUUUG $5^{\prime}$, or a closely related sequence in the case of the promoters for mRNA2, mRNA2-1, and mRNA6 (Table II). The consensus sequences of different coronaviruses are quite similar, though different in length (Table III). Avian infectious bronchitis virus (IBV) has the most divergent sequence, whereas the consensus sequences of the coronaviruses belonging to clusters I and II (Chapter 3) share homology.

Until recently, many basic features of transcription remained unclear due to the lack of an appropriate experimental system. Such issues included the minimal sequence requirements for transcription initiation, the role of basepairing in promoter recognition, and the position(s) at which transcription starts. An important development has been the use of synthetic MHV DI RNAs containing additional intergenic promoter sequences (Makino et al., 1991; van der Most et al., 1994) (Fig. 4). Makino and co-workers (1991) have inserted the mRNA7 promoter and 0.54 kilobase $(\mathrm{kb})$ of its flanking sequences into the genome of DIssF. Transfection of this DI RNA into MHV-infected cells resulted in the synthesis of the DI-derived subgenomic RNA. We have cloned short oligonucleotides (10-18 nt), comprising only the intergenic promoter sequences

TABLE II. Sequences of MHV Intergenic Promoter Sequences

\begin{tabular}{|c|c|}
\hline Promoter & Sequence (on negative strand) \\
\hline mRNA2 & 3'-UUA G A A U A G-5' \\
\hline \multirow{2}{*}{ mRNA2-1 } & $3^{\prime}$ - AUUA - - UUCGA A - 5' \\
\hline & $3^{\prime}$ - AUUA - - UUUGA A - 5' \\
\hline mRNA3 & 3'-AUUAGAUUUG-5' \\
\hline mRNA4 & 3'-UUAGAUUUG -5' \\
\hline mRNA5 & 3'-GAUUAGAUUUG-5' \\
\hline mRNA6 & 3'-UAGAUUAGGUUUGA -5' \\
\hline mRNA7 & 3'-UUAGAUUAGAUUUG A A A U-5' \\
\hline Leader $(+$ strand $)$ & 5'-UGUAGUUUAAAUCUAAUCUAA ACUUUAUAAA-3' \\
\hline
\end{tabular}


TABLE III. Consensus Sequences for Several

Coronaviruses from the Three Clusters ${ }^{a}$

\begin{tabular}{lcr}
\hline Virus & $\begin{array}{c}\text { Antigen } \\
\text { cluster }\end{array}$ & \multicolumn{1}{c}{ Consensus sequence } \\
\hline MHV-A59 & I & 3'-UUAG (A/G)U/U/A)UG-5' $^{\prime}$-UU/U) \\
BCV & I & $3^{\prime}$-AG/A/G)UUUG-5' \\
FIPV & II & $3^{\prime}$-UUGAUUUG-5' \\
TGEV & II & 3'-UUGAUUUG-5' \\
IBV & III & 3'-GAAUUGUU-5' \\
\hline
\end{tabular}

aFrom Spaan et al. (1988).

and no flanking sequences, and found that these sequences alone suffice to direct subgenomic DI RNA synthesis (van der Most et al., 1994).

In this system, promoter strength has been defined as the ratio between the amounts of subgenomic and genomic DI RNAs synthesized in the cell. Insertion of the mRNA7 promoter with $0.54 \mathrm{~kb}$ of flanking sequences into DIssF gave a promoter strength of 0.8 . Extension or deletion of the non-basepaired flanking sequences did not affect promoter strength (Makino and Joo, 1993). Deletions within the intergenic region reduced promoter strength: efficient
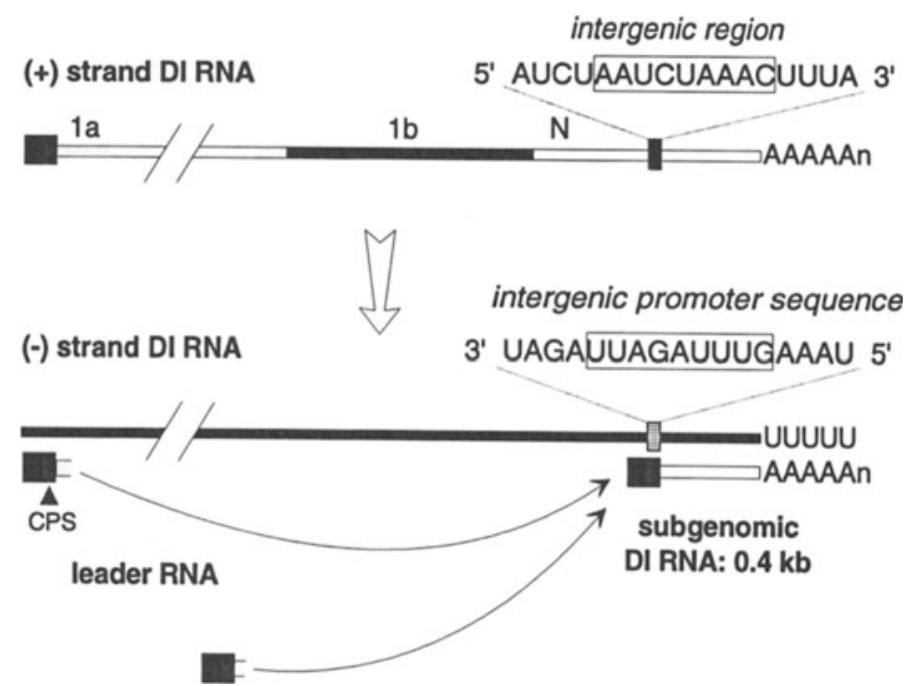

FIGURE 4. Schematic representation of the synthesis of a subgenomic RNA from a DI template. The sequences of the DI that are derived from ORFla (la), ORFIb $(1 \mathrm{~b})$, and the $3^{\prime}$ end of the genome (N) are indicated by different shadings. The DI minus-strand is indicated by a black bar. A 17nucleotide promoter sequence is inserted downstream of the N ORF and is indicated as a dotted box on both the plus- and minus-strands. The complementary promoter sequence (CPS) in the leader is indicated. The 3' UUAGAUUUG 5' consensus sequence and its plus-sense counterpart are boxed. Also indicated are the leader RNAs that originate either from the DI or from the viral genome. 
subgenomic RNA synthesis requires a promoter larger than the partial consensus sequence 3' AGAUUUG 5' (Joo and Makino, 1992; Makino and Joo, 1993; Makino et al., 1991; van der Most et al., 1994). Thus, the intergenic promoter sequence is required for transcription, whereas the flanking sequences are not important.

\section{E. The Control of Subgenomic mRNA Abundance}

The coronavirus subgenomic mRNAs are synthesized in different quantities. For instance, in MHV-infected cells, mRNA7 is synthesized in much greater quantities than mRNA3 (Table I). An important question is whether the determinants that control mRNA abundance reside in the intergenic promoter sequence. Both the sequence of the promoter and the extent of potential basepairing with the leader CPS could determine mRNA levels. For MHV there is a rough correlation between mRNA abundance and the length of the potential duplex. Based on this correlation, it has been proposed that the extent of basepairing controls mRNA abundance (Shieh et al., 1987). However, no such correlation exists for IBV (Konings et al., 1988) and BCV (Hofmann et al., 1993). The relation between the extent of basepairing and mRNA abundance has been tested in our laboratory by inserting the $10 \mathrm{nt}$ mRNA3 and $18 \mathrm{nt}$ mRNA7 promoters into the MIDI genome (van der Most et al., 1994). The "basepairing hypothesis" would predict that the mRNA7 promoter would be stronger than the mRNA3 promoter in this system. On the contrary, we found that the mRNA3 and mRNA7 promoters were of similar strength. In fact, promoter activity was even slightly higher for the mRNA3 promoter. Thus, these data clearly demonstrate that the extent of basepairing does not control mRNA levels.

The role of the specific nucleotide sequence has been studied by mutating single nucleotides in different promoters. Introduction of single substitutions into a $13 \mathrm{nt}$ promoter demonstrated that the sequence of the intergenic promoter can be very flexible (Joo and Makino, 1992). Results from our laboratory show that promoter strength is affected only slightly when a single nucleotide in a 17 $\mathrm{nt}$ promoter or in the $18 \mathrm{nt}$ mRNA7 promoter is mutated (van der Most et al., 1994). In contrast, introduction of the same substitutions into the $10 \mathrm{nt}$ mRNA3 promoter results in a more than tenfold reduction of promoter activity. This could suggest that transcription initiation requires a duplex of a certain minimal stability. Once this condition is met, extending the basepairing does not increase promoter strength. However, some of the substitutions in the 17 or 18 nt promoters did reduce promoter strength, without interrupting the leaderpromoter duplex because G-U basepairs could be formed. This could indicate that recognition of the promoter sequence does not depend on basepairing only. Instead, direct interactions between the polymerase and the promoter could play a role in promoter recognition. Hofmann et al. (1993) came to the same conclusion based on their finding that the leader-body junction of one $\mathrm{BCV}$ mRNA had occurred on the unusual sequence 3 '-CCAUCUG-5' instead of the expected sequence $3^{\prime}$-AGGUUUG-5'. 
The fact that some mutations reduce promoter strength opens the possibility that the levels of certain mRNAs are downregulated because their promoters contain point mutations (e.g., the MHV mRNA6 promoter) (Table II). However, this does not seem to be a general principle. Coronavirus mRNAs are, in general, synthesized at a rate inversely related to their length (Hofmann et al., 1993; Konings et al., 1988; Sethna et al., 1989). This is similar to observations made by French and Ahlquist (1988) who, upon introduction of multiple promoter elements into a synthetic RNA3 of brome mosaic virus, found a gradient of accumulation favoring the smaller mRNAs. Thus, for coronaviruses the transcriptional activity of a promoter may also be (in part) determined by its position on the negative-stranded template. This could be accomplished by premature termination during plus-strand synthesis, i.e., larger RNA molecules are more prone to premature termination of transcription (Konings et al., 1988). Alternatively, premature termination could occur during negative-strand synthesis (Sawicki and Sawicki, 1990). This latter idea is consistent with the transcription model in which a nested set of subgenomic minus strands is synthesized first: larger negative strands are produced in lower quantities because they encounter more transcription attenuators (see Section C) during their synthesis. However, any such model should take into account that, for feline infectious peritonitis virus (FIPV) and TGEV, the shortest mRNAs (RNAs 7 and 6, respectively) are produced in much lower quantities than the next larger mRNA encoding the nucleocapsid protein (de Groot et al., 1987; Sethna et al., 1989). Direct experimental evidence that mRNA abundance is influenced by the presence of additional up- or downstream promoters is not yet available.

\section{F. Heterogeneity of Subgenomic mRNAs}

For MHV, every intergenic promoter contains the sequence 3' UUAGAUUUG 5 ', or a closely related sequence (Table II). The MHV-A59 leader contains two repeats of the 5' UCUAA 3' sequence (Makino et al., 1988b). As mentioned above, the fusion between leader and body sequences occurs within the leader-promoter duplex. For most promoters, a perfect duplex is formed; it is therefore impossible to map the site(s) at which transcription starts. However, some promoters from MHV (e.g., the mRNA6 promoter) and BCV contain mutations, and these mutations are transcribed into the mRNAs (Armstrong et al., 1984; Hofmann et al., 1993). This indicates that transcription started upstream of the mutations. The same phenomenon was observed in an in vitro transcription system (Baker and Lai, 1990). To explain this, Baker and Lai (1990) proposed that 3' terminal sequences of the leader RNA that do not basepair are trimmed and that a hypothetical nuclease recognizes mismatches within the duplex. By using the substitutions in the DI promoters as markers, it was subsequently found that not all mutations were transcribed into the subgenomic mRNAs (Joo and Makino, 1992; van der Most et al., 1994). Mutations at the $5^{\prime}$ end of the promoter were always transcribed, but mutations near the $3^{\prime}$ end gave rise to two populations of mRNAs: mRNAs that had transcribed the mutation and mRNAs that contained the leader sequence (Fig. 5). This sug- 

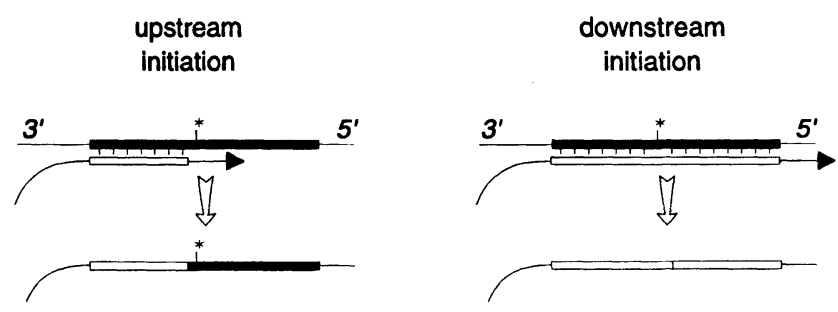

FIGURE 5. The use of markers to map the position(s) of transcription reinitiation. The marker is indicated by an asterisk. Open bars depict leader sequences. Black bars represent the negativestranded template and the sequences that are transcribed from the template.

gested that transcription initiates at multiple-possibly random-sites, presumably resulting from trimming of the leader; mismatch-specific repair does not occur (van der Most et al., 1994).

A different type of heterogeneity has been described for MHV-A59 mRNA6 and MHV-JHM mRNAs 6 and 7 (Armstrong et al., 1984; Baker and Lai, 1990; Makino et al., 1988b; Shieh et al., 1987). For these mRNAs, the number of UCUAA repeats at the leader-body junction is variable. This was also observed for the mRNA7 promoter that had been inserted into MIDI (van der Most et al., 1994). In this system, the leader, which was derived from MHV-A59, contains two pentanucleotide repeats. Two populations of mRNAs were detected: the majority of mRNAs contained two repeats, whereas a minor population had three repeats (Fig. 6). The heterogeneity has been explained by alternative basepairing between these promoters and the leader CPS (Makino et al., 1988b). However, as exemplified in Fig. 6, these promoters contain two (imperfect) repeats of the promoter consensus sequence. Each of these individual "promoter domains" could give rise to a transcript, and these transcripts would differ in the number of repeats. This notion was supported by analyzing mutant promoters in which the ratio between the two transcripts was altered (van der Most et al., 1994).

The number of pentanucleotide repeats in the leader CPS differs for the various MHV strains, e.g., MHV-A59 has two repeats in the leader, whereas the MHV-2c leader contains four repeats (Makino et al., 1988b). During passage of MHV-JHM, the number of pentanucleotide repeats changes from three to two (Makino and Lai, 1989b). The differences in the number of UCUAA repeats of the JHM leader affect the transcription of certain subgenomic mRNAs. For example, transcriptional activity of the mRNA2-1 promoter of MHV-JHM (which encodes the hemagglutinin esterase gene) depends on the number of UCUAA repeats in the leader CPS: mRNA2-1 is only synthesized when the leader CPS contains two UCUAA repeats and not when three repeats are present (Shieh et al., 1989). Additional examples of differential transcription have been described recently (La Monica et al., 1992). There is as yet no explanation for the differential activity of these promoters. This phenomenon has been exploited to demonstrate that the transcription mechanism acts in trans. The mRNA2-1 promoter was inserted into a DI RNA with a three-repeat leader; this 


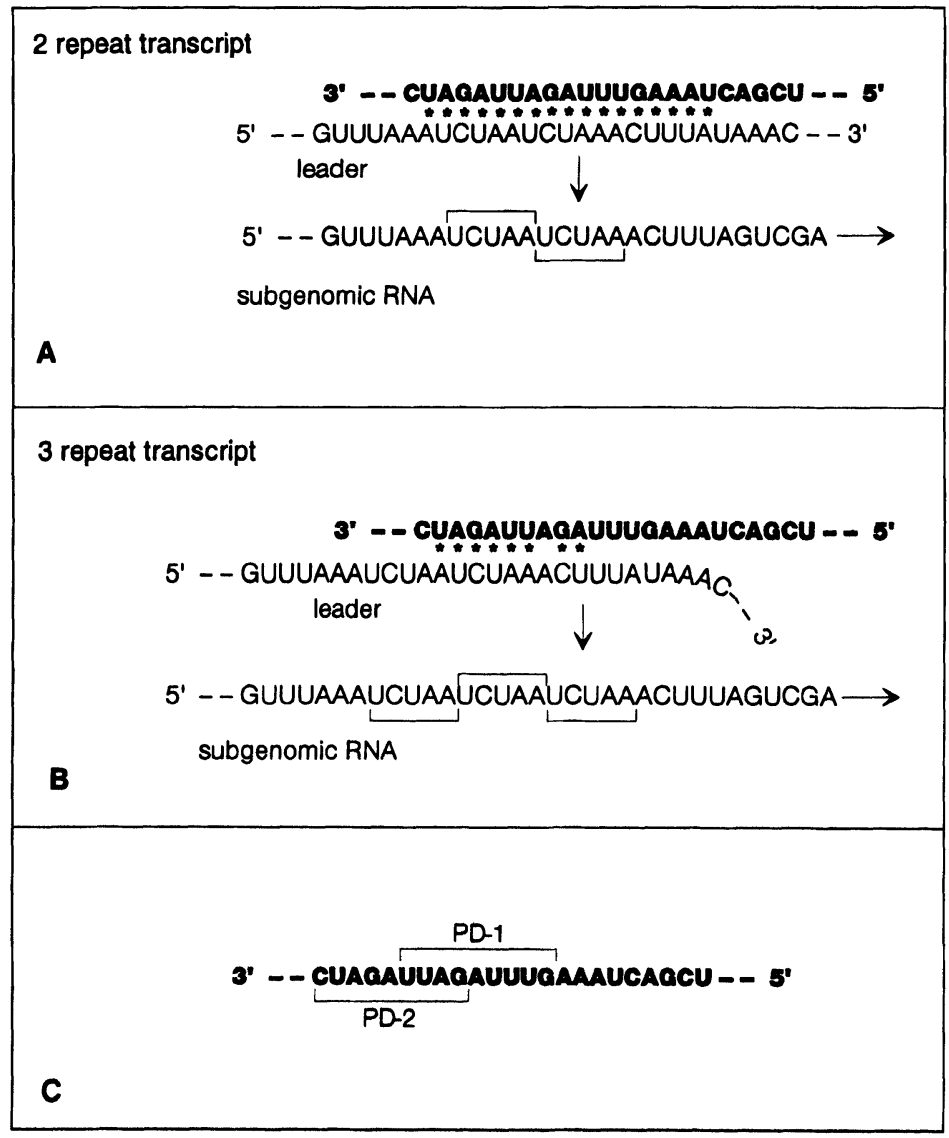

FIGURE 6. Heterogeneity at the leader-body fusion sites of subgenomic RNAs, illustrated for the $17 \mathrm{nt}$ promoter inserted in MIDI. The structure of the DI genome is shown in Fig. 3. The UCUAA repeats in the leader are indicated. Basepairing is indicated by asterisks. (A,B) Synthesis of subgenomic RNAs containing two or three UCUAA repeats. (C) Schematic view of the overlapping promoter domains PD-1 and PD-2.

leader does not support transcription from the inserted promoter. When the helper virus contained a two-repeat leader, a subgenomic DI RNA was synthesized. Because RNA recombination could be excluded, these data indicate that the transcription process occurs in trans (Jeong and Makino, 1994).

\section{RNA RECOMBINATION}

Homologous RNA recombination has been demonstrated for picornaviruses (Cooper, 1968; King et al., 1982), bromoviruses (Allison et a1., 1990; Bujarski and Kaesberg, 1986) and coronaviruses (Lai et al., 1985). During a mixed infection of two MHV strains, RNA recombination occurs at a high frequency (Lai et al., 1985; Makino et al., 1986b). High-frequency RNA recombination 
does not only occur in tissue culture but also in infected mouse brains (Keck et al., 1988a). Based on the analysis of a set of temperature sensitive (ts) mutants, the frequency of recombination for the entire MHV genome has been estimated to be $25 \%$ (Baric et al., 1990). Recombinant murine coronaviruses have been isolated by employing different selection schemes, such as rescue of $t s$ lesions (Baric et al., 1990; Lai et al., 1985; Makino et al., 1986b), resistance to neutralizing monoclonal antibodies (Makino et al., 1987), and rescue of a fusion-negative MHV variant (Keck et al., 1988b). Many of these recombinant viruses had undergone additional recombination events outside the selection markers, again illustrating the high frequency of recombination (reviewed in Lai, 1992). At present, it is not known how widespread homologous RNA recombination is among other coronaviruses. The only other coronavirus for which recombination in vivo has been observed is IBV (Kusters et al., 1990).

Although coronavirus RNA recombination was first thought to take place at certain "hot spots" (Banner et al., 1990), it was later recognized that recombination is almost random and that these previously identified hot spots resulted from selection of recombinant viruses (Banner and Lai, 1991). Coronavirus RNA recombination most likely occurs via template switching: the polymerase and the nascent RNA strand dissociate from the original template and anneal to a different viral RNA molecule after which RNA synthesis continues. Experimental evidence for this copy-choice mechanism has only been obtained for poliovirus recombination (Kirkegaard and Baltimore, 1986), but it is assumed that this mechanism accounts for all examples of homologous RNA recombination (reviewed in Jarvis and Kirkegaard, 1991; Lai, 1992).

The high-frequency RNA recombination that occurs in MHV-infected cells has been exploited to introduce mutations into the viral genome (Koetzner et a1., 1992; Masters et a1., 1994; van der Most et al., 1992). Recombination between the viral genome and co-replicating synthetic DI RNAs yielded recombinant viruses carrying DI sequences. Recombinant viruses were isolated in the absence of any selection or by rescuing a MHV-A59 ts mutant, Albany-4 (van der Most et al., 1992). The ts phenotype of Albany-4 results from a deletion in the $\mathrm{N}$ gene which is repaired by recombination with DI-borne $\mathrm{N}$ sequences. The synthetic RNAs need not be replicating: Albany-4 was also rescued by using a synthetic mRNA7 transcript (Koetzner et al., 1992), which is most probably not amplified on transfection (Brian et al., 1994; Makino et al., 1991; Luytjes and Spaan, unpublished results). However, when a replicating RNA was used, the frequency with which recombinants are isolated is more than 100-fold increased (Masters et al., 1994).

The copy-choice model of recombination proposes that transcription initiates on one template (the "donor" template) and jumps to another template (the "acceptor" template) after which RNA synthesis proceeds (Jarvis and Kirkegaard, 1992). The number of recombinants that are generated depends on the concentrations of both parental RNAs: the concentration of donor template determines the number of initiation events, whereas the frequency of template switching depends on the concentration of acceptor template. This explains why the recombination frequency was increased more than 100 -fold when a 
replicating DI RNA was used instead of the mRNA7 transcript (Masters et al., 1994).

As long as a full-length "infectious" cDNA clone is not available, the only way to introduce genetic changes into a coronavirus genome will be through homologous RNA recombination. A more general applicability of this approach depends on several factors. It will be important to optimize transfection protocols and to investigate which sequences can be cloned into a DI RNA without destroying its capacity to be replicated. Recent results from our laboratory show that a large part of the MHV S gene can be inserted into a DI genome, although the resulting DI RNA replicates less efficiently than the parental DI RNA. One potential limitation of this approach is that introduction of mutations into more internal regions of the genome would require double recombination events. So far, mutations have only been introduced at the $5^{\prime}$ and $3^{\prime}$ regions of the genome. Also, in the case of mutations that decrease viral fitness, screening for recombinant viruses will be tedious. Presumably, such difficulties may be solved by developing efficient screening protocols and by applying selection, e.g., via rescue of $t s$ lesions or by using monoclonal antibodies. Studies to address these issues are undoubtedly in progress.

ACKNOWLEDGMENTS. The authors gratefully acknowledge Dr. Raoul de Groot for stimulating discussions and Dr. Stanley Sawicki for his advice with regard to the role of negative-strand RNAs in transcription.

\section{REFERENCES}

Ahlquist, P., French, R., Janda, M., and Loesch-Fries, L. S., 1984, Multicomponent RNA plant virus infection derived from cloned viral cDNA, Proc. Natl. Acad. Sci. USA 81:7066.

Allison, R., Thompson, C., and Ahlquist, P., 1990, Regeneration of a functional RNA virus genome by recombination between deletion mutants and requirement for cowpea chlorotic mottle virus 3a and coat proteins for systemic infection, Proc. Nat1. Acad. Sci. USA 87:1820.

Andino, R., Rieckhof, G. E., Achacoso, P. L., and Baltimore, D., 1993, Poliovirus RNA synthesis utilizes an RNP complex formed around the 5'-end of viral RNA, EMBO J. 12:3587.

Armstrong, J., Niemann, H., Smeekens, S., Rottier, P., and Warren, G., 1984, Sequence and topology of a model intracellular membrane protein, El glycoprotein, from a coronavirus, Nature 308:751.

Baker, S. C., and Lai, M. M., 1990, An in vitro system for the leader-primed transcription of coronavirus mRNAs, EMBO J. 9:4173.

Baker, S. C., Shieh, C. K., Soe, L. H., Chang, M. F., Vannier, D. M., and Lai, M. M., 1989, Identification of a domain required for autoproteolytic cleavage of murine coronavirus gene A polyprotein, J. Virol. 63:3693.

Baker, S. C., Yokomori, K., Dong, S., Carlisle, R., Gorbalenya, A. E., Koonin, E. V., and Lai, M. M. C., 1993 , Identification of the catalytic sites of a papain-like cysteine proteinase of murine coronavirus, J. Virol. 67:6056.

Banner, L. R., and Lai, M. M., 1991, Random nature of coronavirus RNA recombination in the absence of selection pressure, Virology 185:441.

Banner, L. R., Keck, J. G., and Lai, M. M. C., 1990, A clustering of RNA recombination sites adjacent to a hypervariable region of the peplomer gene of murine coronavirus, Virology 175:548.

Baric, R. S., Stohlman, S. A., Razavi, M. K., and Lai, M. M., 1985, Characterization of leader-related 
small RNAs in coronavirus-infected cells: Further evidence for leader-primed mechanism of transcription, Virus. Res. 3:19.

Baric, R. S., Shieh, C. K., Stohlman, S. A., and Lai, M. M., 1987, Analysis of intracellular small RNAs of mouse hepatitis virus: evidence for discontinuous transcription, Virology 156:342.

Baric, R. S., Fu, K., Schaad, M. C., and Stohlman, S. A., 1990, Establishing a genetic recombination map for murine coronavirus strain A59 complementation groups, Virology 177:646.

Brian, D. A., Chang, R.-Y., Hofmann, M. A., and Sethna, P. B., 1994, Role of subgenomic minu'sstrand RNA in coronavirus replication, Arch. Virol. (Suppl.) 9:173.

Budzilowicz, C. J., Wilczynski, S. P., and Weiss, S. R., 1985, Three intergenic regions of coronavirus mouse hepatitis virus strain A59 genome RNA contain a common nucleotide sequence that is homologous to the $3^{\prime}$ end of the viral mRNA leader sequence, $I$. Virol. 53:834.

Bujarski, J. J., and Kaesberg, P., 1986, Genetic recombination in a multipartite plant virus, Nature 321:528.

Cooper, P. D., 1968, A genetic map of poliovirus temperature-sensitive mutants, Virology 35:584.

de Groot, R. J., ter Haar, R. J., Horzinek, M. C., and van der Zeijst, B. A. M., 1987, Intracellular RNAs of the feline infectious peritonitis coronavirus strain 79-1146, I. Gen. Virol. 68:995.

de Groot, R. J., van der Most, R. G., and Spaan, W. J. M., 1992, The fitness of defective interfering murine coronavirus DI-a and its derivatives is decreased by nonsense and frameshift mutations, $J$. Virol. 66:5898.

Denison, M., and Perlman, S., 1987, Identification of putative polymerase gene product in cells infected with murine coronavirus A59, Virology 157:565.

Denison, M. R., Zoltick, P. W., Leibowitz, J. L., Pachuk, C. J., and Weiss, S. R., 1991, Identification of polypeptides encoded in open reading frame-1b of the putative polymerase gene of the murine coronavirus mouse hepatitis virus-a59, J. Virol. 65:3076.

Denison, M. R., Zoltick, P. W., Hughes, S. A., Giangreco, B., Olson, A. L., Perlman, S., Leibowitz, J. L., and Weiss, S. R., 1992, Intracellular processing of the N-terminal ORF la proteins of the coronavirus MHV-A59 requires multiple proteolytic events, Virology 189:274.

Fosmire, J. A., Hwang, K., and Makino, S., 1992, Identification and characterization of a coronavirus packaging signal, $J$. Virol. 66:3522.

French, R., and Ahlquist, P., 1988, Characterization and engineering of sequences controlling in vitro synthesis of brome mosaic virus subgenomic RNA, J. Virol. 62:2411.

Fuerst, T. R., Niles, E. G., Studier, F. W., and Moss, B., 1986, Eukaryotic transient-expression system based on recombinant vaccinia virus that synthesizes bacteriophage T7 RNA polymerase, Proc. Nat1. Acad. Sci. USA 83:8122.

Furuya, T., and Lai, M. M. C., 1993, Three different cellular proteins bind to complementary sites of the 5 '-end-positive and $3^{\prime}$-end-negative strands of mouse hepatitis virus RNA, $J$. Virol. 67:7215.

Hofmann, M. A., Sethna, P. B., and Brian, D. A., 1990, Bovine coronavirus mRNA replication continues throughout persistent infection in cell culture, J. Virol. 64:4108.

Hofmann, M. A., Chang, R.-Y., Ku, S., and Brian, D. A., 1993, Leader-mRNA junction sequences are unique for each subgenomic mRNA species in the bovine coronavirus and remain so throughout persistent infection, Virology 196:163.

Jacobs, L., Spaan, W. J. M., Horzinek, M. C., and van der Zeijst, B. A. M., 1981, Synthesis of subgenomic mRNAs of mouse hepatitis virus is initiated independently: Evidence from UV transcription mapping, J. Virol. 39:401.

Jarvis, T. C., and Kirkegaard, K., 1991, The polymerase in its labyrinth: Mechanisms and implications of RNA recombination, Trends Genet. 7(6):186.

Jarvis, T. C., and Kirkegaard, K., 1992, Poliovirus RNA recombination: Mechanistic studies in the absence of selection, EMBO $J$. 11:3135.

Jeong, Y. S., and Makino, S., 1992, Mechanism of coronavirus transcription: Duration of primary transcription initiation activity and effects of subgenomic RNA transcription on RNA replication, I. Virol. 66:3339.

Jeong, Y. S., and Makino, S., 1994, Evidence for coronavirus discontinuous transcription, I. Virol. 68:2615.

Joo, M., and Makino, S., 1992, Mutagenic analysis of the coronavirus intergenic consensus sequence, J. Virol. 66:6330.

Keck, J. G., Matsushima, G. K., Makino, S., Fleming, J. O., Vannier, D. M., Stohlman, S. A., and Lai, 
M. M., 1988a, In vivo RNA-RNA recombination of coronavirus in mouse brain, J. Virol. 62:1810.

Keck, J. G., Soe, L. H., Makino, S., Stohlman, S. A., and Lai, M. M., 1988b, RNA recombination of murine coronaviruses: recombination between fusion-positive mouse hepatitis virus A59 and fusion-negative mouse hepatitis virus 2, J. Virol. 62:1989.

Kim, Y.-N., Jeong, Y. S., and Makino, S., 1993a, Analysis of cis-acting sequences essential for coronavirus defective interfering RNA replication, Virology 197:53.

Kim, Y.-N., Lai, M. M. C., and Makino, S., 1993b, Generation and selection of coronavirus defective interfering RNA with large open reading frame by RNA recombination and possible editing, Virology 194:244.

King, A. M., McCahon, D., Slade, W. R., and Newman, J. W., 1982, Recombination in RNA, Cell 29:921.

Kirkegaard, K., and Baltimore, D., 1986, The mechanism of RNA recombination in poliovirus, Cell 47:433.

Koetzner, C. A., Parker, M. M., Ricard, C. S., Sturman, L. S., and Masters, P. S., 1992, Repair and mutagenesis of the genome of a deletion mutant of the coronavirus mouse hepatitis virus by targeted RNA recombination, J. Virol. 66:1841.

Konings, D. A. M., Bredenbeek, P. J., Noten, J. F. H., Hogeweg, P., and Spaan, W. J. M., 1988, Differential premature termination of transcription as a proposed mechanism for the regulation of coronavirus gene expression, Nucleic Acids Res. 16:10849.

Kusters, J. G., Jager, E. J., Niesters, H. G., and van der Zeijst, B. A., 1990, Sequence evidence for RNA recombination in field isolates of avian coronavirus infectious bronchitis virus, Vaccine 8:605.

Lai, C.-J., Zhao, B., Hori, H., and Bray, M., 1991, Infectious RNA transcribed from stably cloned fulllength cDNA of dengue type 4 virus, Proc. Natl. Acad. Sci. USA 88:5139.

Lai, M. M. C., 1990, Coronavirus-organization, replication and expression of genome, Annu. Rev. Microbiol. 44:303.

Lai, M. M. C., 1992, RNA recombination in animal and plant viruses, Microbiol. Rev. 56:61.

Lai, M. M. C., Patton, C. D., and Stohlman, S. A., 1982, Replication of mouse hepatitis virus: Negative-stranded RNA and replicative form RNA are of genome length, $J$. Virol. 44:487.

Lai, M. M., Baric, R. S., Brayton, P. R., and Stohlman, S. A., 1984, Characterization of leader RNA sequences on the virion and mRNAs of mouse hepatitis virus, a cytoplasmic RNA virus, Proc. Natl. Acad. Sci. USA 81:3626.

Lai, M. M., Baric, R. S., Makino, S., Keck, J. G., Egbert, J., Leibowitz, J. L., and Stohlman, S. A., 1985, Recombination between nonsegmented RNA genomes of murine coronaviruses, $J$. Virol. 56:449.

La Monica, N., Yokomori, K., and Lai, M. M., 1992, Coronavirus mRNA synthesis: Identification of novel transcription initiation signals which are differentially regulated by different leader sequences, Virology 188:402.

Lemm, J. A., and Rice, C. M., 1993a, Assembly of functional sindbis virus RNA replication complexes: Requirement for coexpression of $\mathrm{p} 123$ and $\mathrm{p} 34, J$. Virol. 67:1905.

Lemm, J. A., and Rice, C. M., 1993b, Roles of nonstructural polyproteins and cleavage products in regulating sindbis virus RNA replication and transcription, $J$. Virol. 67:1916.

Liao, C. L., and Lai, M. M., 1992, RNA recombination in a coronavirus: Recombination between viral genomic RNA and transfected RNA fragments, J. Virol. 66:6117.

Lin, Y.-J., and Lai, M. M. C., 1993, Deletion mapping of a mouse hepatitis virus defective interfering RNA reveals the requirement of an internal and discontinuous sequence for replication, $J$. Virol. 67:6110.

Lin, Y.-J., Liao, C.-L., and Lai, M. M. C., 1994, Identification of the cis-acting signal for minus-strand RNA synthesis of a murine coronavirus: Implications for the role of minus-strand RNA in RNA replication and transcription, J. Virol. 68:8131.

Makino, S., and Joo, M., 1993, Effect of intergenic consensus sequence flanking sequences on coronavirus transcription, J. Virol. 67:3304.

Makino, S., and Lai, M. M. C., 1989a, High-frequency leader sequence switching during coronavirus defective interfering RNA replication, $J$. Virol. 63:5285.

Makino, S., and Lai, M. M. C., 1989b, Evolution of the $5^{\prime}$-end of genomic RNA of murine coronaviruses during passages in vitro, Virology 169:227. 
Makino, S., Fujioka, N., and Fujiwara, K., 1985, Structure of the intracellular defective viral RNAs of defective interfering particles of mouse hepatitis virus, J. Virol. 54:329.

Makino, S., Stohlman, S. A., and Lai, M. M., 1986a, Leader sequences of murine coronavirus mRNAs can be freely reassorted: Evidence for the role of free leader RNA in transcription, Proc. Nat1. Acad. Sci. USA 83:4204.

Makino, S., Keck, J. G., Stohlman, S. A., and Lai, M. M., 1986b, High-frequency RNA recombination of murine coronaviruses, $J$. Virol. 57:729.

Makino, S., Fleming, J. O., Keck, J. G., Stohlman, S. A., and Lai, M. M., 1987, RNA recombination of coronaviruses: Localization of neutralizing epitopes and neuropathogenic determinants on the carboxyl terminus of peplomers, Proc. Natl. Acad. Sci. USA 84:6567.

Makino, S., Shieh, C. K., Soe, L. H., Baker, S. C., and Lai, M. M., 1988a, Primary structure and translation of a defective interfering RNA of murine coronavirus, Virology 166:550.

Makino, S., Soe, L. H., Shieh, C., and Lai, M. M. C., 1988b, Discontinuous transcription generates heterogeneity at the leader fusion sites of coronavirus mRNAs, $J$. Virol. 62:3870.

Makino, S., Yokomori, K., and Lai, M. M., 1990, Analysis of efficiently packaged defective interfering RNAs of murine coronavirus: Localization of a possible RNA-packaging signal, $J$. Virol. 64:6045.

Makino, S., Joo, M., and Makino, J. K., 1991, A system for study of coronavirus mRNA synthesis: A regulated, expressed subgenomic defective interfering RNA results from intergenic site insertion, J. Virol. 65:6031.

Masters, P. S., Koetzner, C. A., Kerr, C. A., and Heo, Y., 1994, Optimization of targeted RNA recombination and mapping of a novel nucleocapsid gene mutation in the coronavirus mouse hepatitis virus, $J$. Virol. 68:328.

Racaniello, V. R., and Baltimore, D., 1981, Cloned poliovirus complementary DNA is infectious in mammalian cells, Science 214:916.

Rice, C. M., Levis, R., Strauss, J. H., and Huang, H. V., 1987, Production of infectious RNA transcripts from Sindbis virus cDNA clones: Mapping of lethal mutations, rescue of a temperature-sensitive marker, and in vitro mutagenesis to generate defined mutants, $J$. Virol. 61:3809.

Rice, C. M., Grakoui, A., Galler, R., and Chambers, T. J., 1989, Transcription of infectious yellow fever virus RNA from full-length cDNA templates produced by in vitro ligation, New Biol. $1: 285$.

Sawicki, S. G., and Sawicki, D. L., 1990, Coronavirus transcription: Subgenomic mouse hepatitis virus replicative intermediates function in RNA synthesis, $J$. Virol. 64:1050.

Schaad, M. C., and Baric, R. S., 1994, Genetics of mouse hepatitis virus transcription: Evidence that subgenomic negative strands are functional templates, J. Virol. 68:8169.

Sethna, B. P., Hung, S.-L., and Brian, D. A., 1989, Coronavirus subgenomic minus-strand RNAs and the potential for mRNA replicons, Proc. Natl. Acad. Sci. USA 86:5626.

Sethna, P. B., Hofmann, M. A., and Brian, D. A., 1991, Minus-strand copies of replicating coronavirus messenger RNAs contain antileaders, $J$. Virol. 65:320.

Shieh, C. K., Soe, L. H., Makino, S., Chang, M. F., Stohlman, S. A., and Lai, M. M., 1987, The 5 '-end sequence of the murine coronavirus genome: Implications for multiple fusion sites in leaderprimed transcription, Virology 156:321.

Shieh, C. K., Lee, H. J., Yokomori, K., La Monica, N., Makino, S., and Lai, M. M., 1989, Identification of a new transcriptional initiation site and the corresponding functional gene $2 \mathrm{~b}$ in the murine coronavirus RNA genome, J. Virol. 63:3729.

Spaan, W., Delius, H., Skinner, M., Armstrong, J., Rottier, P., Smeekens, S., van der Zeijst, B. A. M., and Siddell, S. G., 1983, Coronavirus mRNA synthesis involves fusion of non-contiguous sequences, $E M B O$ J. 2:1839.

Spaan, W., Cavanagh, D., and Horzinek, M. C., 1988, Coronaviruses: Structure and genome expression, J. Gen. Virol. 69:2939.

Stern, D. F., and Sefton, B. M., 1982, Synthesis of coronavirus mRNAs: Kinetics of inactivation of infectious bronchitis virus RNA synthesis by UV light, $J$. Virol. 42:755.

van der Most, R. G., Bredenbeek, P. J., and Spaan, W. J., 1991, A domain at the 3' end of the polymerase gene is essential for encapsidation of coronavirus defective interfering RNAs, $J$. Virol. 65:3219. 
van der Most, R. G., Heijnen, L., Spaan, W. J. M., and de Groot, R. J., 1992, Homologous RNA recombination allows efficient introduction of site-specific mutations into the genome of coronavirus MHV-A59 via synthetic co-replicating RNAs, Nucleic Acids Res. 20:3375.

van der Most, R. G., de Groot, R. J., and Spaan, W. J. M., 1994, Subgenomic RNA synthesis directed by a synthetic defective interfering RNA of mouse hepatitis virus: A study of coronavirus transcription initiation, J. Virol. 68:3656-3666.

Weiss, S. R., Hughes, S. A., Bonilla, P. J., Turner, J. D., Leibowitz, J. L., and Denison, M. R., 1994, Coronavirus polyprotein processing, Arch. Virol. (Suppl.) 9:349.

Zhang, X., Liao, C.-L. and Lai, M. M. C., 1994, Coronavirus leader RNA regulates and initiates subgenomic mRNA transcription both in trans and in cis, J. Virol. 68:4738. 